\title{
The impact of a standardized micronutrient supplementation on PCOS-typical parameters: a randomized controlled trial
}

\author{
Marlene Hager $^{1} \cdot$ Kazem Nouri $^{1} \cdot$ Martin Imhof $^{2} \cdot$ Christian Egarter $^{1} \cdot$ Johannes Ott $^{1}[$
}

Received: 10 January 2019 / Accepted: 10 May 2019 / Published online: 17 May 2019

(c) The Author(s) 2019

\begin{abstract}
Purpose To evaluate whether a micronutrient supplementation preparation that includes a high amount of omega- 3 unsaturated acids, other anti-oxidants and co-enzyme Q10 would have an impact on specific serum parameters in women with polycystic ovary syndrome (PCOS).

Methods The study was designed as a monocentral, randomized, controlled, double-blinded trial, from June 2017 to March 2018 (Clinical Trials ID: NCT03306745). Sixty women with PCOS were assigned to either the "multinutrient supplementation group" (one unlabeled soft capsule containing omega-3 fatty acids and one unlabeled tablet containing folic acid, selenium, vitamin E, catechin, glycyrrhizin, and co-enzyme Q10, for 3 months) or the "control group" (two unlabeled soft capsules containing $200 \mu \mathrm{g}$ folic acid each, for 3 months). The main outcome parameters were anti-Mullerian hormone $(\mathrm{AMH})$, total testosterone, and androstenedione. In addition, the focus was on luteinizing hormone ( $\mathrm{LH})$, follicle-stimulating hormone (FSH), the LH:FSH ratio, sexual hormone-binding globulin (SHBG), and estradiol.

Results In the multinutrient supplementation group, the LH:FSH ratio $(2.5 \pm 1.1$ versus $1.9 \pm 0.5, p=0.001)$, testosterone $(0.50 \pm 0.19$ versus $0.43 \pm 0.15, p=0.001)$, and AMH $(8.2 \pm 4.2$ versus $7.3 \pm 3.6, p<0.001)$ declined significantly, whereas the other parameters, namely estradiol, LH, FSH, androstenedione, and SHBG remained stable.

Conclusion A micronutrient supplementation that includes omega-3 fatty acids, folic acid, selenium, vitamin E, catechin, glycyrrhizin, and co-enzyme Q10, given for a minimum of 3 months, is beneficial for women with PCOS in terms of PCOSspecific parameters (LH:FSH ratio, serum testosterone and serum AMH).
\end{abstract}

Keywords Micronutrients · Polycystic ovary syndrome $\cdot$ Anti-Mullerian hormone $\cdot$ Testosterone

\section{Introduction}

Polycystic ovary syndrome (PCOS) is a common endocrine disorder that affects about $6-10 \%$ of women of reproductive age [1,2]. In obese patients, lifestyle interventions are generally considered the first-line treatment. Pharmaceutical treatment options include contraceptive pills and hypoglycemic agents [2]. However, contraindications for the oral contraceptive pills are common in this patient population, especially in overweight women, [3] and hypoglycemics often

Johannes Ott

johannes.ott@meduniwien.ac.at

1 Clinical Division of Gynecologic Endocrinology and Reproductive Medicine, Medical University of Vienna, Spitalgasse 23, 1090 Vienna, Austria

2 Department of Obstetrics and Gynecology, Landesklinikum Korneuburg, Korneuburg, Lower Austria, Austria lead to unpleasant side effects $[4,5]$. Thus, it is reasonable that women with PCOS were found to seem unsatisfied with pharmaceutical treatment including oral contraceptives and clomiphene citrate and tended to seek out effective alternative management options [6]. This would obviously include nutritional supplements. However, as reviewed recently, there is insufficient high-quality evidence about the effectiveness of nutritional supplements and herbal medicine for PCOS and its related symptoms. Only some low-quality evidence suggests that PCOS women might benefit from inositols and omega-3 fish oil supplements [7].

However, women with PCOS reveal significantly increased levels of oxidative stress [8] and antioxidants have been claimed to exert positive effects on PCOS [9]. Antioxidants that might positively influence the hormonal profile in PCOS include omega-3 fatty acids [7, 9-14], Vitamin E [11, 13, 14], and selenium [15, 16]. Notably, it has been 
suggested that the anti-inflammatory action of metformin also contributes to the substance's positive effect in PCOS [17].

Notably, meta-analyses question the effects of an omega-3 fatty acid-only treatment $[18,19]$. This raises the question whether single micronutrient supplementations would be of major impact. We, thus, aimed to test the impact of a standardized, multinutrient supplementation on hormonal profile in PCOS women. We chose a micronutrient supplementation preparation that included a high amount of omega-3 unsaturated acids, other anti-oxidants and co-enzyme Q10-the latter had also been shown to improve inflammation and glucose metabolism parameters in previous studies [20, 21] for our prospective, randomized, controlled study.

\section{Materials and methods}

\section{Study population and design}

In a monocentral, prospective randomized controlled double-blinded trial, 60 women with PCOS were included from June 2017 to March 2018. PCOS was diagnosed according to the revised Rotterdam criteria [22, 23]. Participants had to be 19-35 years of age and had to suffer either from oligomenorrhea, defined as an interval of $\geq 60$ days between the last three menstruations, or complete amenorrhoea for at least 90 days. All included women revealed polycystic ovaries on ultrasound. Women who had undergone any kind of PCO-syndrome-related treatment within 3 months before study initiation were excluded. These included metformin, combined oral contraceptives, cortisol therapy, inositol, ovarian drilling, any kind of ovarian stimulation, and in vitro fertilization.

Patients were randomly assigned to one of the following groups: every day, participants received either (i) "PROfertil ${ }^{\circledR}$ female" (Lenus Pharma GesmbH, Seeböckgasse 59, 1160 Vienna), i.e., one unlabeled soft capsule containing omega-3 fatty acids and one unlabeled tablet containing folic acid, selenium, vitamin E, catechin, glycyrrhizin, and coenzyme Q10 (see Table 1 for details on doses; multinutrient supplementation group), or (ii) two unlabeled soft capsules containing $200 \mu \mathrm{g}$ folic acid each or ("Folsäure Kapseln" $400 \mu \mathrm{g}^{\circledR}$, OTC Produktion und Forschung GmbH, Fischergasse 17, 5020 Salzburg; control group).

There were two study-specific consultations: after including the patient (consultation 1: randomization, distribution of drugs and start of study) and after 90-100 days (consultation 2). During consultation 2, unused study blisters were collected and patients were questioned on peculiarities and possible side effects of the micronutrient supplementation used.
Table 1 PROfertil ${ }^{\circledR}$ female composition overview

\begin{tabular}{ll}
\hline Contents & Amount per day \\
\hline Soft capsule & \\
Omega-3 fatty acids & $500 \mathrm{mg}$ \\
Pill & \\
Folic acid & $800 \mu \mathrm{g}$ \\
Selenium & $70 \mu \mathrm{g}$ \\
Vitamin E & $30 \mathrm{mg}$ \\
Green tea extract catechin & $4 \mathrm{mg}$ \\
Glycyrrhizin from licorice extract & $12 \mathrm{mg}$ \\
Co-enzyme Q10 & $30 \mathrm{mg}$ \\
\hline
\end{tabular}

On July 15th, 2017, the study had been approved by the Institutional Review Board of the Medical University of Vienna (IRB number: 1232/2016). The study had been registered on ClinicalTrials.gov (Clinical Trials ID: NCT03306745). All patients signed an informed written consent.

\section{Parameters analyzed}

The main outcome parameters were anti-Mullerian hormone (AMH), total testosterone, and androstenedione. In addition, the focus was on luteinizing hormone (LH), follicle-stimulating hormone (FSH), the LH/FSH ratio, sexual hormone-binding globulin (SHBG), and estradiol. All blood samples were obtained within a maximum of 2 weeks before study initiation (baseline visit) and 90-100 days after beginning supplementation with PROfertil ${ }^{\circledR}$ female or folic acid (3-month follow-up). They were obtained during the early follicular phase visit (cycle days 2-5). If necessary, bleeding was induced using $10 \mathrm{mg}$ of oral dydrogesterone twice a day for 10 days. All serum parameters were determined in the central laboratory of the Medical University of Vienna using commercially available assays.

Moreover, women were explicitly asked for any possible side effects during the study period.

The following additional parameters were also collected as general patient information, i.e., age, body mass index and menstruation history; mean antral follicular count; the HOMA index, calculated as insulin $(\mu \mathrm{U} / \mathrm{mL}) \times$ glucose $(\mathrm{mmol} / \mathrm{L}) / 22.5$ [24], with an abnormal result defined as value $\geq 2$; as well as baseline thyroid-stimulating hormone (TSH) and prolactin levels.

\section{Sample size calculation}

We assumed a reduction of the mean AMH levels by $2 \mathrm{ng} /$ $\mathrm{mL}$ with a standard deviation of $3 \mathrm{ng} / \mathrm{mL}$ at an alpha value of 0.05 and a power of 0.90 . Accordingly, the paired $t$ test would require 26 patients per group. A $15 \%$ drop-out rate 
was assumed. This corresponds to four patients per group. Thus, the final sample size was calculated as 30 patients per group, which led to a total study population of 60 .

\section{Statistical analysis}

Categorical variables are presented as absolute numbers and percentages, numerical variables as median and interquartile range. All numerical variables were normally distributed. Baseline patient characteristics and hormonal patterns as well as the changes in hormonal parameters between the baseline and the 3-month follow-up were analyzed using paired $t$ tests for numerical parameters and the Chi square test or Fisher's exact test for categorical variables. Differences in hormonal patterns between the baseline and the 3 -month follow-up visits were tested using paired $t$ tests. $p$ values of $<0.05$ were considered statistically significant. Statistical analyses were performed using SPSS 24.0 for Windows (SPSS Inc., 1989-2018).
Randomization was performed using nQuery advisorTM Version 7.0.

\section{Results}

Baseline patient and PCOS-specific characteristics of the multinutrient supplementation and the control groups are shown in Table 2. There were no differences between the groups $(p>0.05)$.

Details on outcome in terms of PCOS-specific hormonal patterns are provided in Table 3. No differences between the baseline and the 3-month follow-up visits were found for the control group. In the multinutrient supplementation group, the LH:FSH ratio $(2.5 \pm 1.1$ versus $1.9 \pm 0.5, p=0.001)$, testosterone $(0.50 \pm 0.19$ versus $0.43 \pm 0.15, p=0.001)$, and $\mathrm{AMH}(8.2 \pm 4.2$ versus $7.3 \pm 3.6, p<0.001)$ declined significantly, whereas the other parameters, namely estradiol, LH, FSH, androstenedione, and SHBG remained stable. When
Table 2 Basic patient and PCOS-specific characteristics of the PROfertil ${ }^{\circledR}$ female and the folic acid only groups

\begin{tabular}{llll}
\hline Parameter & $\begin{array}{l}\text { Multinutrient supplementation } \\
\text { group }(n=30)\end{array}$ & $\begin{array}{l}\text { Control group } \\
(n=30)\end{array}$ & $p$ \\
\hline Age (years) & $27.7 \pm 5.7$ & $29.9 \pm 4.9$ & 0.116 \\
BMI $\left(\mathrm{kg} / \mathrm{m}^{2}\right)$ & $26.2 \pm 5.6$ & $25.6 \pm 5.4$ & 0.672 \\
Abnormal HOMA index & $8(26.7)$ & $9(30.0)$ & 1.000 \\
Amenorrhea & $12(40.0)$ & $11(36.7)$ & 1.000 \\
Mean cycle length (days) & $66.1 \pm 21.5$ & $68.4 \pm 18.4$ & 0.653 \\
Mean antral follicular count & $19.3 \pm 4.0$ & $18.7 \pm 1.5$ & 0.722 \\
TSH $(\mu U / \mathrm{mL})$ & $1.5 \pm 0.9$ & $1.7 \pm 0.8$ & 0.300 \\
Estradiol $(\mathrm{pg} / \mathrm{mL})$ & $61.6 \pm 34.5$ & $59.0 \pm 28.1$ & 0.753 \\
Prolactin $(\mathrm{ng} / \mathrm{mL})$ & $17.0 \pm 11.4$ & $13.3 \pm 5.3$ & 0.111 \\
LH $(\mathrm{mU} / \mathrm{mL})$ & $12.9 \pm 6.0$ & $11.1 \pm 6.4$ & 0.261 \\
FSH (mU/mL) & $5.5 . \pm 1.9$ & $5.8 \pm 1.6$ & 0.473 \\
LH:FSH ratio & $2.3 \pm 1.1$ & $2.0 \pm 1.2$ & 0.106 \\
Testosterone $(\mathrm{ng} / \mathrm{mL})$ & $0.50 \pm 0.19$ & $0.43 \pm 0.13$ & 0.137 \\
Androstenedione $(\mathrm{ng} / \mathrm{mL})$ & $3.43 \pm 1.57$ & $3.56 \pm 1.25$ & 0.140 \\
SHBG $(\mathrm{nmol} / \mathrm{L})$ & $47.0 \pm 20.1$ & $48.9 \pm 33.0$ & 0.788 \\
\hline
\end{tabular}

Table 3 Hormonal pattern of PCOS patients in the course of the study

\begin{tabular}{|c|c|c|c|c|c|c|}
\hline \multirow[t]{2}{*}{ Parameter } & \multicolumn{3}{|c|}{ Multinutrient supplementation group $(n=28)$} & \multicolumn{3}{|c|}{ Folic acid only group $(n=28)$} \\
\hline & Baseline visit & 3-month follow-up & $p$ & Baseline visit & 3-month follow-up & $p$ \\
\hline Estradiol (pg/mL) & $60.71 \pm 39.60$ & $57.18 \pm 26.23$ & 0.201 & $59.61 \pm 29.02$ & $57.50 \pm 23.07$ & 0.545 \\
\hline $\mathrm{LH}(\mathrm{mU} / \mathrm{mL})$ & $13.2 \pm 6.1$ & $10.7 \pm 3.6$ & 0.011 & $11.2 \pm 6.5$ & $10.0 \pm 5.3$ & 0.172 \\
\hline $\mathrm{FSH}(\mathrm{mU} / \mathrm{mL})$ & $5.5 \pm 1.9$ & $5.8 \pm 1.8$ & 0.241 & $5.9 \pm 1.6$ & $5.2 \pm 1.4$ & 0.038 \\
\hline LH:FSH ratio & $2.5 \pm 1.1$ & $1.9 \pm 0.5$ & 0.001 & $2.0 \pm 1.2$ & $2.0 \pm 0.9$ & 0.744 \\
\hline Testosterone $(\mathrm{ng} / \mathrm{mL})$ & $0.50 \pm 0.19$ & $0.43 \pm 0.15$ & 0.001 & $0.43 \pm 0.13$ & $0.44 \pm 0.12$ & 0.475 \\
\hline Androstenedione (ng/mL) & $3.36 \pm 1.61$ & $3.17 \pm 1.40$ & 0.282 & $3.51 \pm 1.20$ & $3.53 \pm 0.98$ & 0.918 \\
\hline SHBG (nmol/L) & $46.4 \pm 20.2$ & $48.3 \pm 19.2$ & 0.252 & $44.2 \pm 27.3$ & $47.1 \pm 26.7$ & 0.223 \\
\hline AMH (ng/mL) & $8.2 \pm 4.2$ & $7.3 \pm 3.6$ & $<0.001$ & $8.0 \pm 4.1$ & $8.0 \pm 4.0$ & 0.968 \\
\hline
\end{tabular}


comparing the baseline to follow-up changes in hormonal patterns between the multinutrient supplementation and the control groups, significant differences were found for FSH, the LH:FSH ratio, testosterone, and AMH $(p<0.05$; Table 4). None of the patients reported any side effects.

\section{Comment}

This prospective, randomized study on PCOS women demonstrated that the use of a multinutrient supplementation containing omega- 3 fatty acids, folic acid, selenium, vitamin E, catechin, glycyrrhizin, and co-enzyme Q10 led to a significant reduction in the LH:FSH ratio, testosterone and $\mathrm{AMH}$, when compared to the use of $400 \mathrm{mg}$ folic acid alone. These data support previous reports about beneficial effects of micronutrients, at least of those with an antioxidant activity, on PCOS-specific hormonal profiles [7, 9-16].

It has to be mentioned that previous meta-analyses questioned the effects of a supplementation with omega-3 fatty acids only $[18,19]$. It seems obvious that the positive effect which was seen in the present study might be due to the multinutrient approach. However, this approach makes it impossible to attribute the treatment's positive impact to one of the ingredients. The included ingredients might affect the specific parameters of PCOS via different mechanisms. While omega-3 fatty acids, selenium, vitamin E, and also co-enzyme Q10, known to scavenge free radicals and inhibit lipid and protein oxidation [25], might have acted as antioxidants and thereby likely influenced the proinflammatory PCOS state [9], licorice with its active component glycyrrhizin has been shown to affect androgen metabolism, probably via blocking the activity of $3-\beta$-hydroxysteroid dehydrogenase (3HSD), 17-hydroxysteroid dehydrogenase (17HSD) and 17-20 lyase [26-33] and stimulation of the aromatase activity [31]. Notably, a lowering effect on ovarian androgens has already been reported for licorice [33], even in PCOS women [32]. Last not least, catechin has been reported to decrease testosterone production, at least in male rats. While the authors concluded that the demonstrated decrease in androgens were due to changes in enzymatic activities [34], an antioxidant mechanism cannot be excluded for our study [35].

Thus, one could raise the issue of the used preparation's composition. PROfertil ${ }^{\circledR}$ female was chosen, since it was a standardized and commercially available multinutrient supplementation. Taken all these considerations together, the fact that the exact impact of each of the ingredients cannot be assessed in detail needs to be considered a study limitation.

Notably, there was one other important difference between the two groups. Since many women with PCOS suffer from infertility, the recommended standard folic acid supplementation of a daily dose of $400 \mu \mathrm{g}$ [36] was provided to the control group. However, the multinutrient supplementation group received twice the dose. Notably, high-dose folic acid supplementation has been suggested to lead to an improvement in inflammatory factors, biomarkers of oxidative stress [37] as well as metabolic parameters in PCOS women [38]. This might have also contributed to the beneficial effects observed in the multinutrient supplementation group.

Our results must be interpreted with care due to the following additional considerations: although the study seems well randomized (Table 2), patients could have searched for the actual look of the PROfertil ${ }^{\circledR}$ female soft capsules and the pills, for example on the internet. Since the look of the commercially available preparation differs from that of the folic acid capsules, patients could have identified the control medications. This kind of blinding was not according to standards and could have introduced some kind of bias. Moreover, despite the small sample size to achieve, the study period was quite long. Empirically, this was a result of the delay in standardized PCOS treatment for 90-100 days. Accordingly, repeatedly women were not willing to participate. In addition, no data about patients' dietary habits were collected. Last not least, one could argue that the power was only sufficient to test an AMH difference of $2 \mathrm{ng} /$ $\mathrm{mL}$, whereas a difference of only $1 \mathrm{ng} / \mathrm{mL}$ was found in our study, which still reached statistical significance. However, in the sample size calculation, a much higher AMH standard
Table 4 Mean changes of hormonal patterns between baseline and 3-month follow-up in the PROfertil ${ }^{\circledR}$ female and the folic acid-only groups

\begin{tabular}{lccc}
\hline Parameter & $\begin{array}{l}\text { Multinutrient supplementation } \\
\text { group }(n=28)\end{array}$ & Control group $(n=28)$ & $p$ \\
\hline Estradiol $(\mathrm{pg} / \mathrm{mL})$ & $-6.36 \pm 25.65$ & $-2.11 \pm 18.19$ & 0.477 \\
LH $(\mathrm{mU} / \mathrm{mL})$ & $-2.5 \pm 4.8$ & $-1.3 \pm 4.7$ & 0.349 \\
FSH $(\mathrm{mU} / \mathrm{mL})$ & $0.4 \pm 1.6$ & $-0.8 \pm 1.9$ & 0.018 \\
LH:FSH ratio & $-0.6 \pm 0.9$ & $-0.1 \pm 0.9$ & 0.016 \\
Testosterone (ng/mL) & $-0.06 \pm 0.09$ & $0.01 \pm 0.07$ & 0.001 \\
Androstenedione $(\mathrm{ng} / \mathrm{mL})$ & $-0.12 \pm 1.03$ & $0.01 \pm 0.73$ & 0.587 \\
SHBG $(\mathrm{nmol} / \mathrm{L})$ & $1.8 \pm 8.3$ & $-2.5 \pm 10.6$ & 0.094 \\
AMH $(\mathrm{ng} / \mathrm{mL})$ & $-1.0 \pm 1.2$ & $0.0 \pm 1.1$ & 0.004 \\
\hline
\end{tabular}


deviation of $3 \mathrm{ng} / \mathrm{mL}$ had been expected in contrast to the actual standard deviation of about $1.2 \mathrm{ng} / \mathrm{mL}$, which explains why the lower mean difference in AMH was still found to be significant.

In conclusion, our data suggest that a micronutrient supplementation that includes omega-3 fatty acids, folic acid, selenium, vitamin E, catechin, glycyrrhizin, and co-enzyme Q10, given for a minimum of 3 months, is beneficial for women with PCOS in terms of PCOS-specific parameters, namely the LH:FSH ratio, serum testosterone and serum AMH. Although the clinical relevance of these results can be challenged, the presented date should encourage future studies about the influence of micronutrients on PCOS, hopefully including data about specific symptoms and infertility treatment outcomes as additional outcome parameters.

Acknowledgments Open access funding provided by Medical University of Vienna.

Author contributions All the authors contributed to the writing process of the manuscript and approved the final version. $\mathrm{MH}$ : acquisition of data, statistical analyses, drafting the article and revising it for intellectual content, and final approval of the version to be published. $\mathrm{KN}$ : the project's and the manuscript's conception and design, drafting the article and revising it for intellectual content, and final approval of the version to be published. MI: the project's and the manuscript's conception and design, drafting the article and revising it for intellectual content, and final approval of the version to be published. CE: the project's and the manuscript's conception and design, acquisition of data, drafting the article and revising it for intellectual content, and final approval of the version to be published. JO: the project's and the manuscript's conception and design, acquisition of data, statistical analyses, drafting the article and revising it for intellectual content, and final approval of the version to be published.

Funding This trial received funding from Lenus Pharma GesmbH.

\section{Compliance with ethical standards}

Conflict of interest M. Imhof and J. Ott received speaker honorarium for lecturing from Lenus Pharma GesmbH.

Ethical approval All the procedures performed in studies involving human participants were in accordance with the ethical standards of the institutional and/or national research committee and with the 1964 Helsinki Declaration and its later amendments or comparable ethical standards. On July 15th, 2017, the study was approved by the Institutional Review Board of the Medical University of Vienna (IRB number: 1232/2016). The study was registered on ClinicalTrials.gov (Clinical Trials ID: NCT03306745).

Informed consent Informed consent was obtained from all individual participants included in the study.

Open Access This article is distributed under the terms of the Creative Commons Attribution 4.0 International License (http://creativeco mmons.org/licenses/by/4.0/), which permits unrestricted use, distribution, and reproduction in any medium, provided you give appropriate credit to the original author(s) and the source, provide a link to the Creative Commons license, and indicate if changes were made.

\section{References}

1. Azziz R, Woods KS, Reyna R, Key TJ, Knochenhauer ES, Yildiz BO (2004) The prevalence and features of the polycystic ovary syndrome in an unselected population. J Clin Endocrinol Metab 89(6):2745-2749

2. McCartney CR, Marshall JC (2016) Clinical practice. Polycystic ovary syndrome. N Engl J Med 375(1):54-64

3. Diamanti-Kandarakis E, Baillargeon JP, Iuorno MJ, Jakubowicz DJ, Nestler JE (2003) A modern medical quandary: polycystic ovary syndrome, insulin resistance, and oral contraceptive pills. J Clin Endocrinol Metab 88(5):1927-1932

4. Legro RS, Barnhart HX, Schlaff WD, Carr BR, Diamond MP, Carson SA, Steinkampf MP, Coutifaris C, McGovern PG, Cataldo NA, Gosman GG, Nestler JE, Giudice LC, Leppert PC, Myers ER, Network Cooperative Multicenter Reproductive Medicine (2007) Clomiphene, metformin, or both for infertility in the polycystic ovary syndrome. N Engl J Med 356(6):551-566

5. Tang T, Lord JM, Norman RJ, Yasmin E, Balen AH (2012) Insulin-sensitising drugs (metformin, rosiglitazone, pioglitazone, D-chiro-inositol) for women with polycystic ovary syndrome, oligo amenorrhoea and subfertility. Cochrane Database Syst Rev CD003053

6. Sills ES, Perloe M, Tucker MJ, Kaplan CR, Genton MG, Schattman GL (2001) Diagnostic and treatment characteristics of polycystic ovary syndrome: descriptive measurements of patient perception and awareness from 657 confidential self-reports. BMC Womens Health 1(1):3

7. Arentz S, Smith CA, Abbott J, Bensoussan A (2017) Nutritional supplements and herbal medicines for women with polycystic ovary syndrome; a systematic review and meta-analysis. BMC Complement Altern Med 17(1):500

8. Murri M, Luque-Ramírez M, Insenser M, Ojeda-Ojeda M, Escobar-Morreale HF (2013) Circulating markers of oxidative stress and polycystic ovary syndrome (PCOS): a systematic review and meta-analysis. Hum Reprod Update 19(3):268-288

9. Amini L, Tehranian N, Movahedin M, Ramezani Tehrani F, Ziaee S (2015) Antioxidants and management of polycystic ovary syndrome in Iran: a systematic review of clinical trials. Iran J Reprod Med 13(1):1-8

10. Mejia-Montilla J, Reyna-Villasmil E, Domínguez-Brito L, Naranjo-Rodríguez C, Noriega-Verdugo D, Padilla-Samaniego M, Vargas-Olalla V (2018) Supplementation with omega-3 fatty acids and plasma adiponectin in women with polycystic ovary syndrome. Endocrinol Diabetes Nutr 65(4):192-199

11. Jamilian M, Shojaei A, Samimi M, Afshar Ebrahimi F, Aghadavod E, Karamali M, Taghizadeh M, Jamilian H, Alaeinasab S, Jafarnejad S, Asemi Z (218) The effects of omega-3 and vitamin E co-supplementation on parameters of mental health and gene expression related to insulin and inflammation in subjects with polycystic ovary syndrome. J Affect Disord 229:41-47.

12. Mirmasoumi G, Fazilati M, Foroozanfard F, Vahedpoor Z, Mahmoodi S, Taghizadeh M, Esfeh NK, Mohseni M, Karbassizadeh H, Asemi Z (2018) The effects of flaxseed oil omega-3 fatty acids supplementation on metabolic status of patients with polycystic ovary syndrome: a randomized, double-blind placebocontrolled trial. Exp Clin Endocrinol Diabetes 126(4):222-228

13. Ebrahimi FA, Samimi M, Foroozanfard F, Jamilian M, Akbari H, Rahmani E, Ahmadi S, Taghizadeh M, Memarzadeh MR, Asemi Z (2017) The effects of omega-3 fatty acids and vitamin E cosupplementation on indices of insulin resistance and hormonal parameters in patients with polycystic ovary syndrome: a randomized, double-blind placebo-controlled trial. Exp Clin Endocrinol Diabetes 125(6):353-359 
14. Rahmani E, Samimi M, Ebrahimi FA, Foroozanfard F, Ahmadi S, Rahimi M, Jamilian M, Aghadavod E, Bahmani F, Taghizadeh M, Memarzadeh MR, Asemi Z (2017) The effects of omega-3 fatty acids and vitamin E co-supplementation on gene expression of lipoprotein(a) and oxidized low-density lipoprotein, lipid profiles and biomarkers of oxidative stress in patients with polycystic ovary syndrome. Mol Cell Endocrinol 439:247-255

15. Mohammad Hosseinzadeh F, Hosseinzadeh-Attar MJ, Yekaninejad MS, Rashidi B (2016) Effects of selenium supplementation on glucose homeostasis and free androgen index in women with polycystic ovary syndrome: a randomized, double blinded, placebo controlled clinical trial. J Trace Elem Med Biol 34:56-61

16. Razavi M, Jamilian M, Kashan ZF, Heidar Z, Mohseni M, Ghandi Y, Bagherian T, Asemi Z (2016) Selenium supplementation and the effects on reproductive outcomes, biomarkers of inflammation, and oxidative stress in women with polycystic ovary syndrome. Horm Metab Res 48(3):185-190

17. Sirmans SM, Weidman-Evans E, Everton V, Thompson D (2012) Polycystic ovary syndrome and chronic inflammation: pharmacotherapeutic implications. Ann Pharmacother 46:403-418

18. Sadeghi A, Djafarian K, Mohammadi H, Shab-Bidar S (2017) Effect of omega-3 fatty acids supplementation on insulin resistance in women with polycystic ovary syndrome: meta-analysis of randomized controlled trials. Diabetes Metab Syndr 11(2):157-162

19. Hajishafiee M, Askari G, Iranj B, Ghiasvand R, Bellissimo N, Totosy de Zepetnek J, Salehi-Abargouei A (2016) The effect of $n-3$ polyunsaturated fatty acid supplementation on androgen status in patients with polycystic ovary syndrome: a systematic review and meta-analysis of clinical trials. Horm Metab Res 48(5):281-289

20. Rahmani E, Jamilian M, Samimi M, Zarezade Mehrizi M, Aghadavod E, Akbari E, Tamtaji OR, Asemi Z (2018) The effects of coenzyme Q10 supplementation on gene expression related to insulin, lipid and inflammation in patients with polycystic ovary syndrome. Gynecol Endocrinol 34(3):217-222

21. Samimi M, Zarezade Mehrizi M, Foroozanfard F, Akbari H, Jamilian M, Ahmadi S, Asemi Z (2017) The effects of coenzyme Q10 supplementation on glucose metabolism and lipid profiles in women with polycystic ovary syndrome: a randomized, double-blind, placebo-controlled trial. Clin Endocrinol (Oxf) 86(4):560-566

22. Thessaloniki ESHRE/ASRM-Sponsored PCOS Consensus Workshop Group (2008) Consensus on infertility treatment related to polycystic ovary syndrome. Fertil Steril 89:505-522

23. Thessaloniki ESHRE/ASRM-Sponsored PCOS Consensus Workshop Group (2008) Consensus on infertility treatment related to polycystic ovary syndrome. Hum Reprod 23(3):462-477

24. Ehrmann DA, Barnes RB, Rosenfield RL, Cavaghan MK, Imperial J (1999) Prevalence of impaired glucose tolerance and diabetes in women with polycystic ovary syndrome. Diabetes Care 22:141-146

25. Papucci L, Schiavone N, Witort E, Donnini M, Lapucci A, Tempestini A, Formigli L, Zecchi-Orlandini S, Orlandini G, Carella G, Brancato R, Capaccioli S (2003) Coenzyme q10 prevents apoptosis by inhibiting mitochondrial depolarization independently of its free radical scavenging property. J Biol Chem 278(30):28220-28228

26. Armanini D, Bonanni G, Palermo M (1999) Reduction of serum testosterone in men by licorice. N Engl J Med 341:1158

27. Armanini D, Bonanni G, Mattarello MJ, Fiore C, Sartorato P, Palermo M (2003) Licorice consumption and serum testosterone in healthy man. Exp Clin Endocrinol Diabetes 111:341-343

28. Barbieri RL, Rein MS, Hornstein MD, Ryan KJ (1988) Rat Leydig cell and granulosa cells 17-keto reductase activity: subcellular localization and substrate specificity. Am J Obstet Gynecol 159:1564-1569

29. Bogovich K, Payne AH (1980) Purification of rat testicular microsomal 17-ketosteroid reductase. Evidence that 17-ketosteroid reductase and 17 beta-hydroxysteroid dehydrogenase are distinct enzymes. J Biol Chem 255:5552-5559

30. Latif SA, Conca TJ, Morris DJ (1990) The effects of the licorice derivative, glycyrrhetinic acid, on hepatic 3 alpha- and 3 betahydroxysteroid dehydrogenases and 5 alpha- and 5 beta-reductase pathways of metabolism of aldosterone in male rats. Steroids 55:52-58

31. Sakamoto K, Wakabayashi K (1988) Inhibitory effect of glycyrrhetinic acid on testosterone production in rat gonads. Endocrinol Jpn 35:333-342

32. Takahashi K, Yoshino K, Shirai T, Nishigaki A, Araki Y, Kitao M (1988) Effect of traditional herbal medicine (Shakuyaku-KanzoTo) on testosterone secretion in patients with polycystic ovary syndrome detected by ultrasound. Acta Obstet Gynaecol Jpn 40:789-792

33. Takeuchi T, Nishii O, Okamura T, Yaginuma T (1991) Effect of paeoniflorin, glycyrrhizin and glycyrrhetinic acid on ovarian androgen production. Am J Chin Med 19:73-78

34. Yu PL, Pu HF, Chen SY, Wang SW, Wang PS (2010) Effects of catechin, epicatechin and epigallocatechin gallate on testosterone production in rat leydig cells. J Cell Biochem 110(2):333-342

35. Frei B, Higdon JV (2003) Antioxidant activity of tea polyphenols in vivo: evidence from animal studies. J Nutr 133:3275-3384

36. Farahi N, Zolotor A (2013) Recommendations for preconception counseling and care. Am Fam Physician 88:499-506

37. Bahmani F, Karamali M, Shakeri H, Asemi Z (2014) The effects of folate supplementation on inflammatory factors and biomarkers of oxidative stress in overweight and obese women with polycystic ovary syndrome: a randomized, double-blind, placebo-controlled clinical trial. Clin Endocrinol (Oxf) 81(4):582-587

38. Asemi Z, Karamali M, Esmaillzadeh A (2014) Metabolic response to folate supplementation in overweight women with polycystic ovary syndrome: a randomized double-blind placebo-controlled clinical trial. Mol Nutr Food Res 58(7):1465-1473

Publisher's Note Springer Nature remains neutral with regard to jurisdictional claims in published maps and institutional affiliations. 